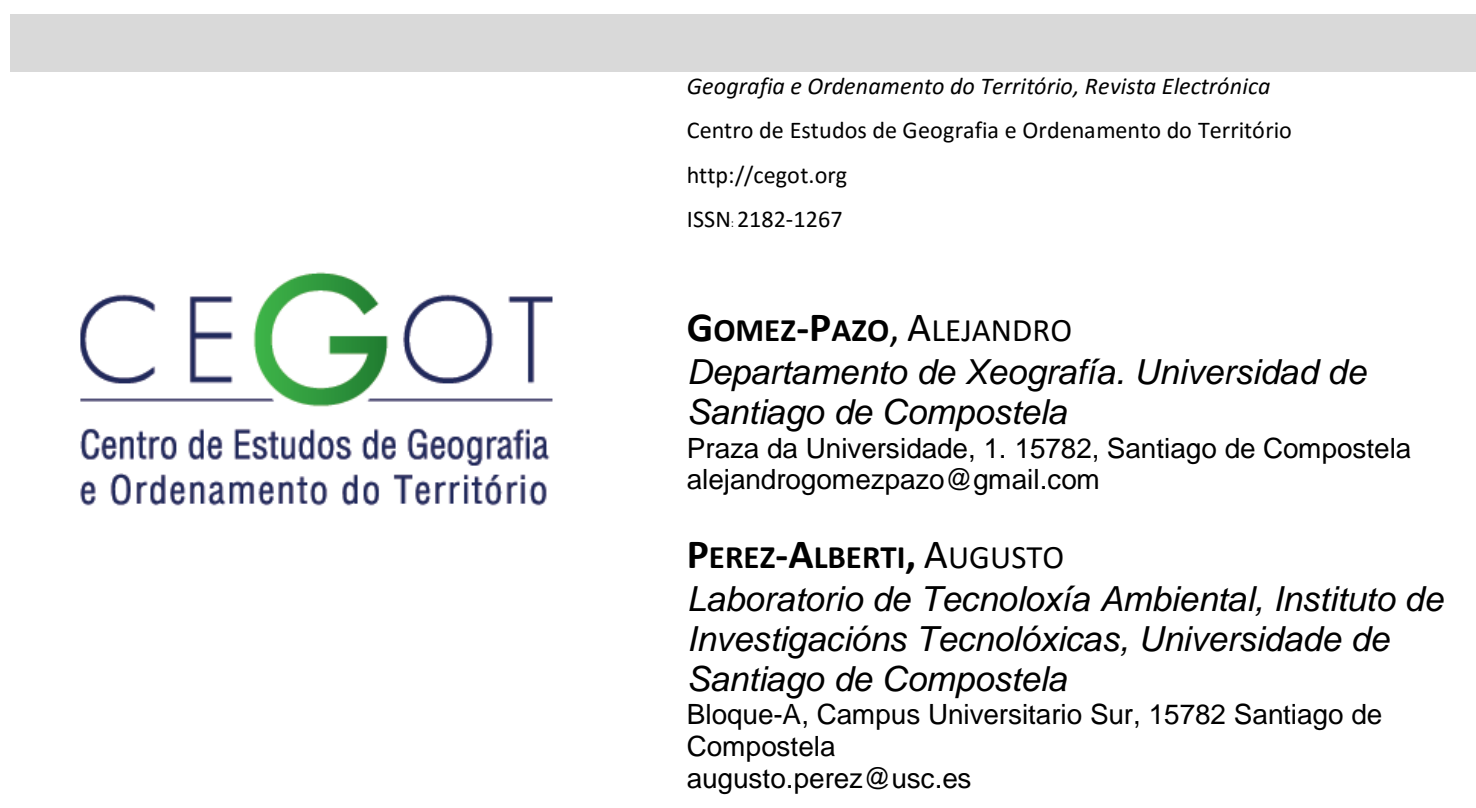

\title{
Aplicación de los SIG en la clasificación morfológica de los tipos de costa: el ejemplo de las Illas Cíes (Galicia, NO de la Península Ibérica)
}

Aplicação dos SIG na classificação morfológica dos tipos de costa: o exemplo das Ilhas Cíes (Galícia, NO da Península Ibérica)

Application of GIS in the morphological classification of types of coast: the example of Cíes Islands (Galicia, NW Iberian Peninsula)

Referência:Gomez-Pazo, Alejandro; Pérez-Alberti, Augusto (2016). Aplicación de los SIG en la clasificación morfológica de los tipos de costa: el ejemplo de las Illas Cíes (Galicia, NO de la Península Ibérica). Revista de Geografia e Ordenamento do Território (GOT), n. 9 (junho). Centro de Estudos de Geografia e Ordenamento do Território, p. 161-185, dx.doi.org/10.17127/got/2016.9.008

\section{RESUMEN}

La preocupación por las regiones costeras ha sido muy importante a lo largo de la historia. En los últimos años, con la evolución experimentada por los SIG ha surgido una gran oportunidad de profundizar en su mejor conocimiento. La presente investigación se ha centrado en buscar una aproximación metodológica semiautomática a las costas rocosas que ayude a poder caracterizar mejor las costas rocosas. Para ello se han seleccionado las Illas Cíes. Se han usado ortofotografías y datos LIDAR, a partir de los cuales se ha generado información del terreno de 0,5 m de resolución. Su combinación ha permitido diferenciar 10 tipos de costa singularizados por su asociación a plataformas litorales, acumulaciones de bloques en su base o distintos perfiles topográficos, así como por enmarcarse en sectores acantilados o de sistemas playa-duna.

Palabras clave: SIG, costa, geomorfología litoral, Islas Cíes. 


\section{RESUMO}

A preocupação pelas regiões costeiras tem sido muito importante ao longo da história. Nos últimos anos, com a evolução experimentada pelos SIG, surgiu uma grande oportunidade para aprofundar a sua compreensão. Esta pesquisa centrou-se numa abordagem metodológica semi-automática para a classificação das costas rochosas que ajudam a caracterizar melhor as costas rochosas. Para este feito foram selecionadas as llhas Cies. Usaram-se ortofotos e dados LIDAR, com os quais se geraram informações da superfície com $0,5 \mathrm{~m}$ de resolução. A sua combinação permitiu individualizar 10 tipos de costa em ligação com as suas plataformas costeiras associação, as acumulações de blocos na sua base ou associadas a diferentes perfis topográficos, assim como, enquadradas em sectores escarpados ou sistemas de praia-duna.

Palavras-chave: GIS, costa, geomorfologia costeira, Ilhas Cíes.

\section{ABSTRACT}

The concern for coastal regions has been very important throughout history. In recent years, with the evolution experienced by the SIG has emerged a great opportunity to deepen your understanding. This research has focused on search a semi-automatic methodological approach to the rocky shores that help to better characterize the rocky ecosystems. For this purpose, the Illas Cíes have been selected. It have used orthophotos and LIDAR data, from which it has generated information from the field of $0.5 \mathrm{~m}$ resolution. Their combination has allowed 10 types of coast singled out by his association with coastal platforms, accumulations of blocks in your base, or different topographic profiles, as well as framed in sectors cliffs or beach-dune systems.

Keywords: GIS, coast, coastal geomorphology, Cíes Islands.

\section{Introducción}

El interés por el conocimiento de las zonas costeras viene siendo una cuestión fundamental para los investigadores a lo largo de la historia (Carter, 1998; Bird, 2008; Woodroffe, 2003) y dentro de ellas, por su menor grado de conocimiento, las costas rocosas (Emery y Kuhn, 1982; Trenhaile, 1987; Trenhaile et al., 1999). En cualquier caso, la comprensión de su dinámica ayuda a una mejor planificación y gestión así como a predecir cualquier evento catastrófico (Pérez-Alberti et al., 2002; Del Río y Gracia, 2007, 2009; Del Río, Gracia y Benavente, 2009). En la actualidad, el grado de conocimiento de la franja litoral supera las necesidades existentes en el pasado, en relación con el acelerado cambio global, que augura 
un aumento del nivel medio del mar (IPCC, 2013). Por lo tanto, poder conocer el aumento potencial del nivel medio del mar en los distintos puntos del planeta es muy importante para la seguridad de la población y para el mantenimiento de las infraestructuras (D'Ercole et al., 2011). Es igualmente clave el conocimiento de los riesgos a los que están sometidas las zonas costeras en función de diversos factores como la diversidad litológica, las formas topográficas o la influencia oceánica (Del Río y Gracia, 2009). Unido a este interés por los sistemas costeros se debe destacar la importancia de los avances logrados por los Sistemas de Información Geográfica en los últimos tiempos, transformándose en una herramienta indispensable, para el análisis de la realidad territorial (Dikau, 1989; Green y King, 2003; Nelson et al., 2015; Terranova et al. 2009; Ferraris, Firpo y Pazzaglia, 2012; Gao et al., 2013), permitiendo conseguir un alto nivel de detalle, que otorga a los estudios y sus resultados una mayor seguridad y precisión.

Los avances tanto en las herramientas SIG como, especialmente el acceso a fuentes de información, como Modelos Digitales del Terreno y ortofotografías, ha facilitado su uso (Lin y Oguchi, 2006; Kasei et al., 2009; Kawabata y Bandibas, 2009; Ebert et al., 2011; Pereira y Pérez-Alberti, 2013; Pérez-Alberti, 2014) y favorecido la puesta en marcha de nuevas investigaciones, y más concretamente en las costas rocosas (Trenhaile et al., 1999; PérezAlberti et al., 2011; Pérez-Alberti et al., 2012; Pérez-Alberti et al., 2013; Pérez-Alberti y Trenhaile, 2015).

Teniendo en cuenta lo anterior se puede entender la importancia de conocer las principales características que determinan la forma y el comportamiento de cada segmento de costa. Por ello, mediante la presente investigación se ha tratado de realizar, con la ayuda de las herramientas SIG, una clasificación de tipos de costa que permita llegar a un mejor entendimiento de sus dinámicas y predecir su posible evolución. Para ello se han usado ortofotografías y datos LIDAR $^{1}$ procedentes del $\mathrm{PNOA}^{2}$, a partir de los cuales se han generado Modelos Digitales del Terreno (MDT o MDS).

\footnotetext{
${ }^{1}$ (Light Detection and Ranging). Se trata de un sensor activo que emite pulsos de luz que interactúan con la atmósfera o el suelo de distinto modo según el tipo de elemento con el que se encuentre. (Chuvieco, 2010)

${ }^{2}$ Plan Nacional de Ortofotografía Aéreas http://goo.gl/kBntUe [Consulta: 28/12/2015]
} 


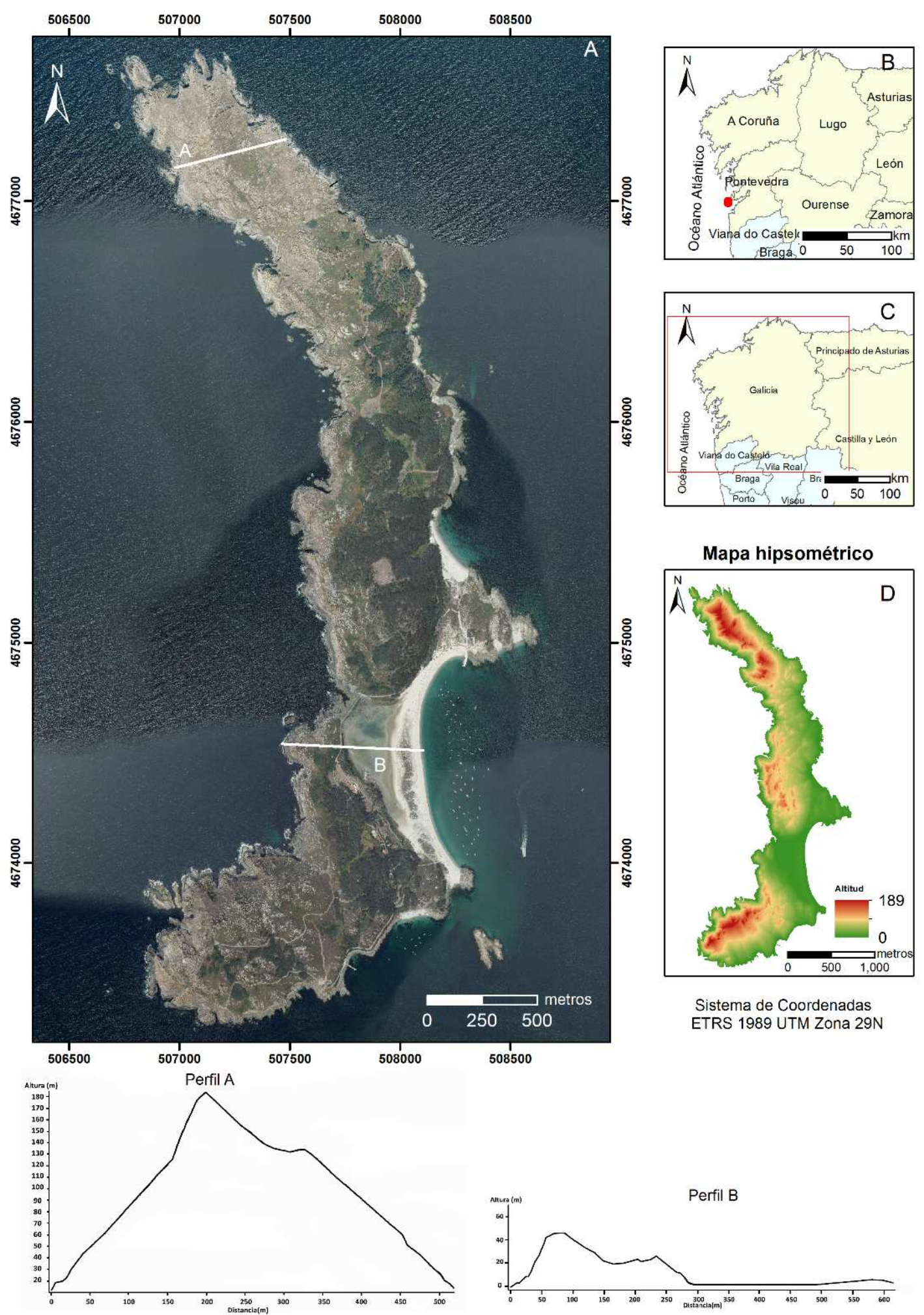

Figura 1 - Mapa de localización y perfiles topográficos.

El área de estudio se sitúa en el archipiélago de las Illas Cíes, en Galicia (Figura 1), y más concretamente en dos de sus islas, Faro y Monteagudo, en donde se ha hecho una 
clasificación de los tipos de costa buscando una metodología que pudiera ser extrapolable a otras zonas costeras. El archipiélago de las Illas Cíes se encuentra en el noroeste de la Península Ibérica, más concretamente en Galicia, región que cuenta con alrededor de 2.100 $\mathrm{km}$ de costa, teniendo en cuenta las diversas islas (POLGalicia, 2010). Se trata de un territorio con una gran heterogeneidad en lo que a tipos de costa se refiere, siendo los contrastes muy acusados en pequeños tramos litorales en los que se puede asistir a una sucesión de costas acantiladas y amplios arenales (Pérez-Alberti, 1986; Pérez-Alberti y Blanco-Chao, 2005). La zona de estudio a la que se ciñe este trabajo comprende la totalidad de las islas de Monteagudo y Faro, que forman la parte más extensa del archipiélago, con cerca de 300 hectáreas (Mouriño Lourido et al., 2002).

\section{Caracterización del área de estudio}

Las Illas Cíes están situadas en la boca de la Ría de Vigo (Figura 1). Su posición juega un importante papel como limitador de los intercambios entre el interior de la ría y el océano, definiendo en buena medida la evolución del archipiélago, que cuenta con dos vertientes muy diferenciadas: una occidental más abrupta donde se alcanzan las mayores altitudes y pendientes y otra oriental que está dominada por la costa baja, con pendientes reducidas en la mayor parte y con una presencia destacada de arenales, que no existen en la vertiente más expuesta al océano (Figura 1, perfiles A y B).

Litológicamente dominan los granitos de dos micas de facies de grano medio intensamente fracturados, lo que está directamente relacionado con la configuración de las Cíes en general y en particular con su modelado de detalle. El origen del archipiélago se retrotrae al de las rías y del relieve litoral del NO de la Península Ibérica, íntimamente ligado a la tectónica como ya defendía Pannekoek (1970). Al mismo tiempo no se pueden olvidar en ningún caso la acción erosiva tanto marina como fluvial, que ha tenido un papel complementario, al igual que ha ocurrido con los procesos fríos (Casal Vila, 2003). En el caso concreto del área de estudio la etapa más importante para su formación se sitúa en el Terciario inferior, durante el Paleógeno, hace entre 65 y 24 millones de años, momento en 
el que se generó una gran falla que se alarga desde el sur de Aveiro (Portugal) hasta el norte de las Illas Cíes, con una longitud de más de 300 kilómetros. Este sistema de fracturación está ligado con strike-slips faults (de Vicente et al., 2011) y ha generado formas sigmoides que se pueden apreciar con gran claridad en distintos puntos de la costa y a diferentes escalas.

Su combinación provocó la existencia de zonas de debilidad que fueron aprovechadas por la erosión marina para abrir corredores que separan la costa acantilada. Algunos de estos han sido colmatados por sedimentos durante los periodos fríos del cuaternario (Pérez-Alberti et al., 2009). Su posterior destrucción ha provocado la existencia de playas de bloques (coídos) en la base y acantilados verticales de remate plano, cuya presencia se ciñe esencialmente a la vertiente occidental del archipiélago.

Por su carácter insular la zona presenta características climáticas diferentes a la zona continental más próxima de Vigo y Península do Morrazo. Se puede considerar como un clima atlántico subhúmedo, con unas diferencias térmicas muy reducidas a lo largo del año con una temperatura media de $13.8^{\circ} \mathrm{C}$ y precipitaciones moderadas con una media de 877 $\mathrm{mm}$, que son casi nulas en los meses estivales, produciéndose el $70 \%$ entre los meses de octubre y marzo (Carballeira et al., 1983) que pueden llegar a representar en algunos años la mitad de las que caen en el continente (Costas, 2008) hecho que, además de relacionarlo con la condición de insularidad mencionada anteriormente, también se debe ligar a la baja altitud del archipiélago, no superando los 192 metros.

Por su parte la dirección del viento es mayoritariamente de componente norte o nornordeste, en el punto $\operatorname{SIMAR}^{3}$ más próximo, teniendo en cuenta los valores anuales. Diferenciando por estaciones destaca la presencia de la componente S-SO durante el invierno, que desaparece casi en su totalidad durante el verano. El oleaje que llega al archipiélago es predominantemente de componente O-NO en periodos anuales, pero al igual que para los vientos existen variaciones estacionales. Durante el invierno el oleaje es más claramente de componente oeste (Figura 2).

\footnotetext{
${ }^{3}$ Punto SIMAR 1044069 localizado al oeste de la isla Norte o de Monteagudo.
} 

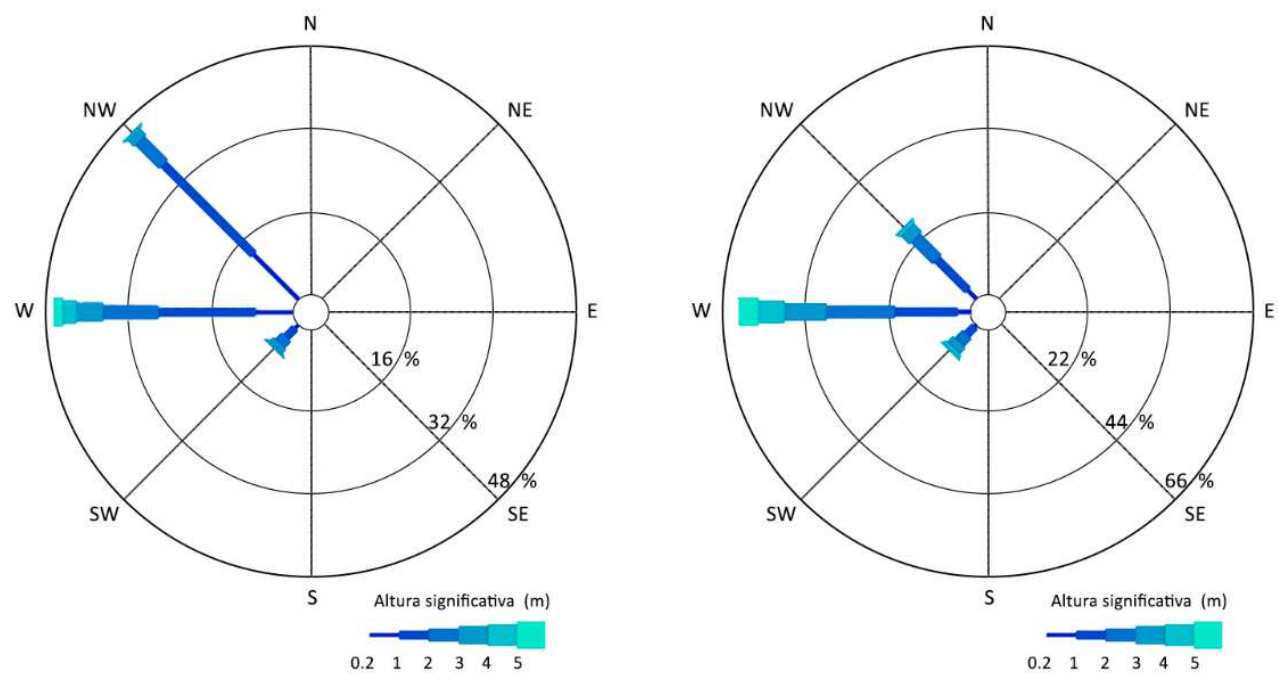

Figura 2 - Rosas de oleaje anual (izquierda) e invernal (derecha) $)^{4}$

En este punto también se registran las alturas máximas de ola, dato muy importante de cara a entender las zonas en las que la erosión es mayor, que se encuentran principalmente en la fachada occidental y el norte de la Illa de Monteagudo, sectores que por su disposición reciben de forma más directa las olas de mayor altitud durante el periodo invernal. A este respecto cabe destacar que durante el invierno, en el período 1958-2015, el 31 \% de las olas superan los 3 metros de altura significativa, cifra que se reduce en las demás estaciones, siendo aproximadamente del $14 \%$ en primavera y otoño y casi insignificantes en el verano. En términos anuales y teniendo en consideración los datos anteriores, se puede decir que un $20 \%$ de las olas registradas superan los 3 metros de altura significativa. Otro elemento fundamental para caracterizar el litoral es el régimen mareal dominante en el mismo, siendo en este caso de carácter mesomareal y con una periodicidad semidiurna, es decir, dos pleamares y dos bajamares cada 24 horas (Rosón et al., 2008).

En cuanto al nivel de protección del archipiélago de las lllas Cíes se debe destacar que fueron declaradas Parque Nacional en el año 2002, junto a otros archipiélagos gallegos formando el Parque Nacional Marítimo-Terrestre das Illas Atlánticas de Galicia, pero ya desde el año 1980 gozaban de la categoría de Parque Natural.

Se trata de un territorio que ha tenido una influencia humana relativamente importante, a diferencia de lo que se podría pensar, y cuyas modificaciones en el terreno tienen un gran impacto en la actualidad, sobre todo las realizadas en el último siglo. Esta influencia queda

\footnotetext{
${ }^{4}$ Datos de Puertos del Estado recogidos en el punto SIMAR 1044069 para el período 1958-2015 con un intervalo de 3 horas y considerando como invierno el periodo de diciembre a febrero.
} 
patente de un modo muy perceptible en la vegetación arbórea del archipiélago, que tras las repoblaciones de la década de 1950 por parte del ICONA ${ }^{5}$, está dominada por eucaliptos (Eucalyptus globulus) y pinos (Pinus pinaster), reduciéndose en buena medida las especies propias del lugar. A ellos hay que unirle la construcción de las infraestructuras que se pueden encontrar en la actualidad en las Cíes, como son los muelles, destacando sobre todo el de Rodas por su mayor actividad y el dique que une la isla de Monteagudo y de Faro. Tras su construcción este dique sufrió una serie de reparaciones y modificaciones en la década de 1940 a causa de su deterioro y en la actualidad aún continúa marcando la dinámica del lagoon (Figura 3).
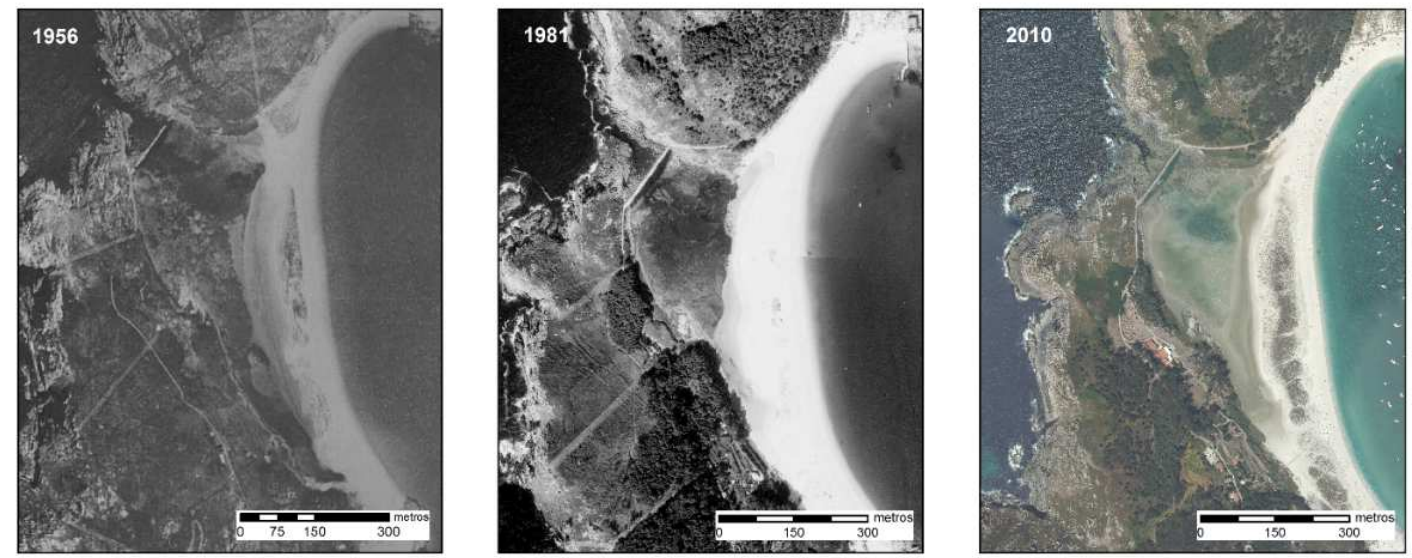

Figura 3 - Aspecto del lagoon en 1956, 1981 y 2010.

Se trata de una pieza fundamental de cara a poder entender la evolución futura que puede vivir la zona del sistema de Rodas, siendo el manejo de la dinámica del lagoon uno de los elementos de interés para la realización de diferentes estudios.

El ser humano también ha producido cambios reseñables en el comportamiento de los sistemas arenosos, como en el caso de Rodas con la extracción de arena entre 1950 y 1970 (Costas, 2006) y más recientemente con el aumento del número de visitantes a las islas y el uso que realizan de los sistemas arenosos.

\footnotetext{
${ }^{5}$ Instituto para la Conservación de la Naturaleza. Organismo administrativo español que desaparece en 1991 con la asunción de la mayoría de sus competencias por las Comunidades Autónomas.
} 


\section{Metodología}

Para la caracterización de los tipos de costa se han empleado los programas ArcGIS 10.3 (licencia de la USC) y SAGA 2.1.2 (Conrad et al., 2015). El material usado han sido las ortofotografías del vuelo americano, 1956; del vuelo interministerial del 1981; del SIGPAC, 2003 y del PNOA (2010). Esta última es la más recientes de las disponibles para la zona de estudio y en la que se basa primordialmente este estudio. Además de los materiales anteriores también se ha empleado el Modelo Digital del Terreno de 5 metros (MDT05LIDAR) y los archivos LAS del LIDAR. Toda esta información ha sido extraída del Centro de Descargas del Instituto Geográfico Nacional ${ }^{6}$. Para poder conseguir un mayor nivel de detalle en el análisis y por lo tanto llegar a una mayor exactitud en la clasificación, se optó por realizar un Modelo Digital del Terreno para toda la isla a partir de los datos de la nube de puntos LIDAR. Para tal fin se empleó el software SAGA y se realizó un modelo digital con una resolución de 0,5 m. La utilización de estos archivos (LAS) se debe a la gran calidad del MDT que se puede construir (Pereira et al., 2013). La diferencia entre el modelo del IGN de 5 metros de resolución y este se aprecia con gran claridad, como se puede ver al realizar el sombreado de ambos archivos (Figura 4).
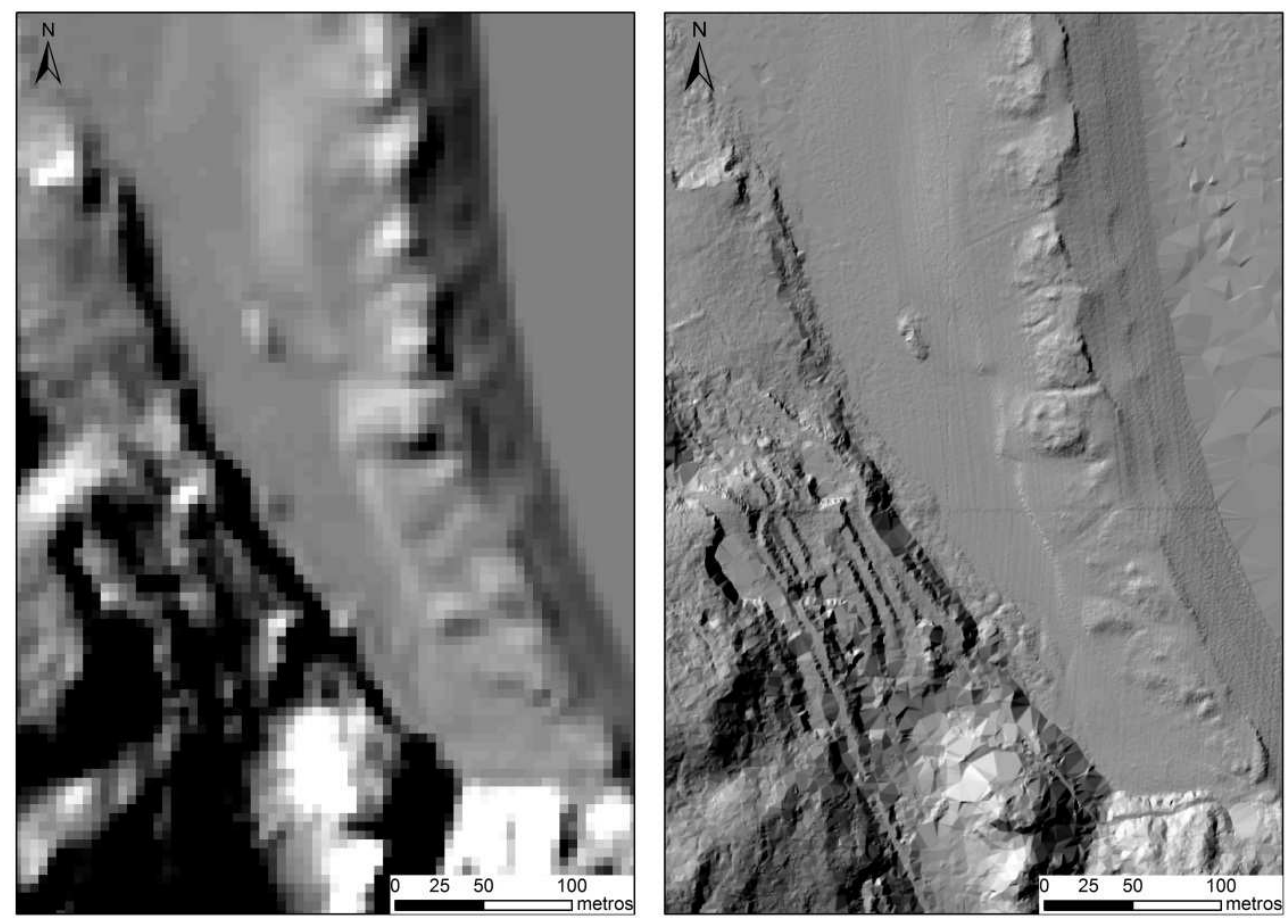

Figura 4 - Localización de la zona y comparación MDT con pixel $5 \mathrm{~m}$ y MDT con pixel de $0.5 \mathrm{~m}$.

\footnotetext{
${ }^{6}<$ http://centrodedescargas.cnig.es/CentroDescargas/index.jsp> (Consulta a: 25/11/2015).
} 
El procedimiento seguido en SAGA se limitó a la selección únicamente de los datos de la nube de puntos LIDAR que hiciesen referencia al terreno, según la clasificación de los mismos realizada por el IGN que acompaña a los archivos descargables. Una vez definidos estos valores se depuraron todas aquellas zonas, sobre todo en el mar, que pudiesen llevar a errores de altura, como consecuencia de los rebotes. Después de unir todos los archivos que forman el área de trabajo se realizó su unión para posteriormente construir el MDT mediante la función Triangulation dentro del entorno Gridding de SAGA. Con el archivo ya finalizado y exportado se prosiguió su análisis en ArcGIS, elaborando distintas capas de Altitud $(A)$ y Pendiente $(P)$ a partir del MDT de 0.5 metros. De este modo se consiguieron dos capas diferenciadas que se clasificaron de la manera que aparece reflejada en tabla 1.

\begin{tabular}{|c|c|}
\hline Altitud (A)(metros) & Pendiente (P) \\
\hline $0-15$ & $3-15^{\circ}$ \\
\hline $16-30$ & $16-30^{\circ}$ \\
\hline $31-50$ & $31-50^{\circ}$ \\
\hline $51-80$ & $51-85^{\circ}$ \\
\hline $81-120$ & \\
\hline $121-189$ & \\
\hline
\end{tabular}

Tabla 1 - Intervalos seleccionados para la altura y la pendiente del frente costero.

En relación con la altura se generó una clasificación en 6 categorías, en función del rango altitudinal existente. Su distribución se ha basado en el intento de que todos los grupos pudieran ser característicos y que no hubiera escalones vacíos en contraposición con rangos extremadamente amplios, en los que pudiesen aparecer un número de registros elevado.

En cuanto a la pendiente se optó por una clasificación en 5 categorías, una vez analizada la diversidad de pendientes existentes en la zona de estudio mediante los datos del MDT, la 
ortofotografía aérea y del trabajo de campo. Esta diversidad queda patente en la diferencia entre pendientes muy bajas y zonas que pueden llegar a los $80^{\circ}$. Con estas agrupaciones se ha buscado conocer las diferencias existentes entre los distintos sectores que se encadenan en la franja costera destacando la división entre los dos últimos rangos. Dicha división ha buscado una caracterización idónea para el archipiélago, ya que las zonas con pendientes superiores a los $30^{\circ}$ representaban un área demasiado amplia dentro de la zona de estudio, por lo que de no realizarse la agrupación de zonas con pendiente superiores a los $50^{\circ}$ los resultados visuales de esta variable serian poco representativos.

Tras una primera agrupación de los tipos de costa a partir de cada una de estas variables de formas separada, que no proporcionó ningún dato relevante, se realizó una conversión a formato poligonal y posteriormente se interseccionaron ambas para lograr un único shape que agrupara las características tanto de altura como de pendiente en un mismo polígono.

Con los datos resultantes y la ayuda de la ortofotografía se procedió a la delimitación de lo que se consideró como zona costera, para la posterior delimitación de cada agrupación. La línea utilizada para tal fin ha sido la del tope superior del acantilado, o cliff top, definida en el lugar donde la ruptura de pendiente muestra con claridad una diferenciación entre la costa/acantilado propiamente dicho y la zona interior. Este tipo de delimitación acostumbra usarse en el caso de los acantilados verticales por la mayor facilidad para su definición, pero para este estudio se consideró que esta aproximación era la más idónea, dentro de la dificultad que entraña su definición en una costa como la de las Cíes. La utilización de la ortofotografía ha sido el último recurso, para marcar el cliff top, otorgándole en todos los casos un mayor peso a los datos objetivos de altura y pendiente, que se mencionaron con anterioridad.

Una vez definida la línea de costa se elaboró una clasificación de los tipos de costa en función de las variables anteriores, dando como resultado 15 categorías. En una primera aproximación no se alcanzó el nivel de detalle, ni la diferenciación entre las distintas agrupaciones deseado, por lo que a partir de los 15 grupos mencionados se realizaron una serie de perfiles topográficos, a lo largo de toda la costa del archipiélago, con el fin de poder caracterizar mejor los distintos tipos de costa. Con este procedimiento el número de tipos 
de costa se vio reducido a 10 , un número bastante manejable y que muestra con claridad las diferencias existentes en las Illas Cíes.

\section{Resultados}

A través de los procedimientos que se han mostrado en el apartado anterior, se llegó a la clasificación final de 10 tipos de costa para el archipiélago de las Illas Cíes, cuya distribución se puede ver en la (Figura 5). Esta caracterización engloba desde los tipos de mayor pendiente como son los 1 y 2, que destacan en la zona más expuesta a la acción marina, hasta la categoría 10 que representa la costa baja, dominada por los arenales de la vertiente oriental, donde la erosión causada por el océano es menor (Tabla 2). Todas las características de los diferentes tipos de costa que figuran en la (Tabla 2) se han definido a partir del análisis conjunto de las distintas variables empleadas en este estudio, y mencionadas con anterioridad, como son la pendiente, la altura y los perfiles topográficos de las distintas zonas analizadas, pudiendo comprobar su localización en la Figura 5. Para lograr realizar una mención de las principales características de cada una de las tipologías se ha analizado con más detalle la totalidad de la superficie ocupada por las mismas, para de este modo llegar a una caracterización que tenga en cuenta las formas más destacas de cada sector del archipiélago. 

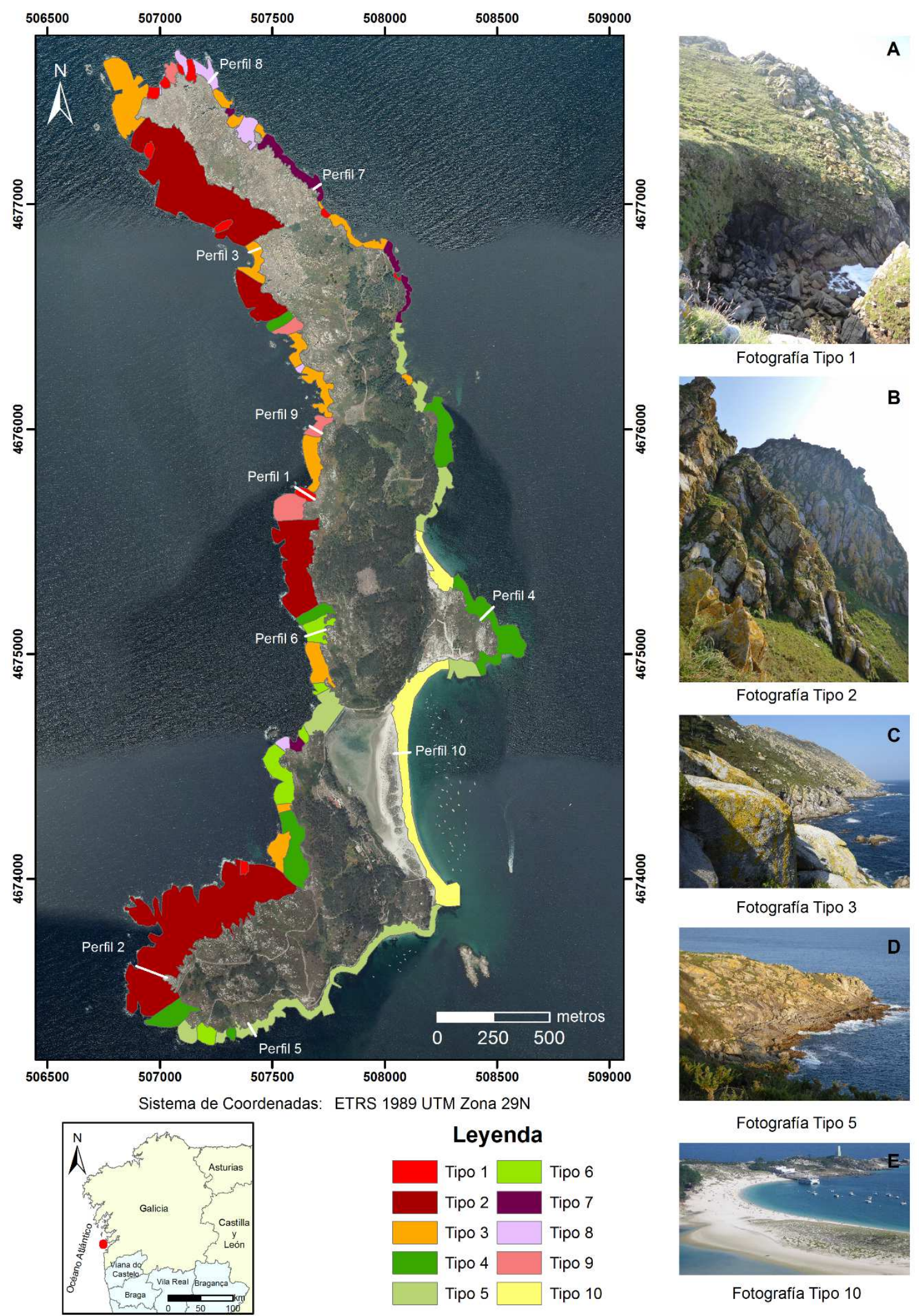

\section{Leyenda}

\begin{tabular}{|l|l|}
\hline Tipo 1 & \\
\hline \\
\hline Tipo 2 & Tipo 6 \\
\hline Tipo 3 & Tipo 7 \\
\hline Tipo 4 & Tipo 8 \\
\hline Tipo 5 & Tipo 9 \\
\hline
\end{tabular}

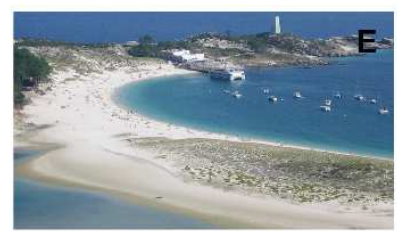

Fotografia Tipo 10

Figura 5 - Mapa de distribución de los tipos de costa, la localización de los cortes topográficos y ejemplos de los tipos de costa más destacados. 


\begin{tabular}{|c|c|c|c|c|}
\hline Tipo & Altura & Pendiente & Características & Situación \\
\hline 1 & $>16 \mathrm{~m}$ & $>50$ & Acantilados verticales & $\begin{array}{c}\text { Fachada occidental y extremo } \\
\text { norte de Monteagudo }\end{array}$ \\
\hline 2 & $>80 \mathrm{~m}$ & $>50$ & Acantilados de mayor altitud & Vertiente occidental \\
\hline 3 & $<80 \mathrm{~m}$ & $>15$ & $\begin{array}{l}\text { Frecuente presencia de coídos } \\
\text { en la base de los acantilados }\end{array}$ & $\begin{array}{l}\text { Norte de las islas y fachada } \\
\text { occidental }\end{array}$ \\
\hline 4 & $\begin{array}{l}>80 \mathrm{~m} \\
<80 \mathrm{~m}\end{array}$ & $\begin{array}{l}15-30 \\
3-15\end{array}$ & $\begin{array}{c}\text { Agrupación compleja según } \\
\text { perfil }\end{array}$ & $\begin{array}{c}\text { Mayormente en la fachada } \\
\text { oriental }\end{array}$ \\
\hline 5 & $<80 \mathrm{~m}$ & $3-30$ & Perfil compuesto & $\begin{array}{c}\text { Zona del dique, fachada orienta } \\
\text { y extremo sur }\end{array}$ \\
\hline 6 & $30-80 m$ & $15-30$ & $\begin{array}{l}\text { Perfil marcado por la etapa } \\
\text { evolutiva de la plataforma }\end{array}$ & $\begin{array}{c}\text { Vertiente occidental próxima al } \\
\text { dique }\end{array}$ \\
\hline 7 & $<30 \mathrm{~m}$ & $30-50$ & $\begin{array}{l}\text { Con plataforma en la base del } \\
\text { acantilado }\end{array}$ & Zona norte vertiente oriental \\
\hline 8 & $<30 \mathrm{~m}$ & $30-50$ & $\begin{array}{l}\text { Sin plataforma en la base de los } \\
\text { acantilados }\end{array}$ & $\begin{array}{c}\text { Extremo norte de la vertiente } \\
\text { oriental }\end{array}$ \\
\hline 9 & $30-80 m$ & $30-50$ & $\begin{array}{c}\text { Sin acumulaciones en la base el } \\
\text { acantilado }\end{array}$ & $\begin{array}{c}\text { Vertiente occidental de la isla } \\
\text { norte }\end{array}$ \\
\hline 10 & $<15 \mathrm{~m}$ & $>3$ & Costa baja/arenales & Vertiente oriental \\
\hline
\end{tabular}

Tabla 2 - Características de los 10 tipos de costa.

El tipo 1 de costa definido se corresponde con los acantilados de mayor pendiente (Figura 6), pudiendo llegar a superar los $80^{\circ}$ en algunas zonas, característica que hace que sólo se encuentre en aquellas zonas donde la influencia marina es mayor. Se trata de áreas en las que predominan los procesos erosivos relacionados con la llegada de olas de mayor altura del período invernal, frecuentemente de componente O-NO, las cuales impiden la acumulación de sedimentos en la base de los acantilados. 

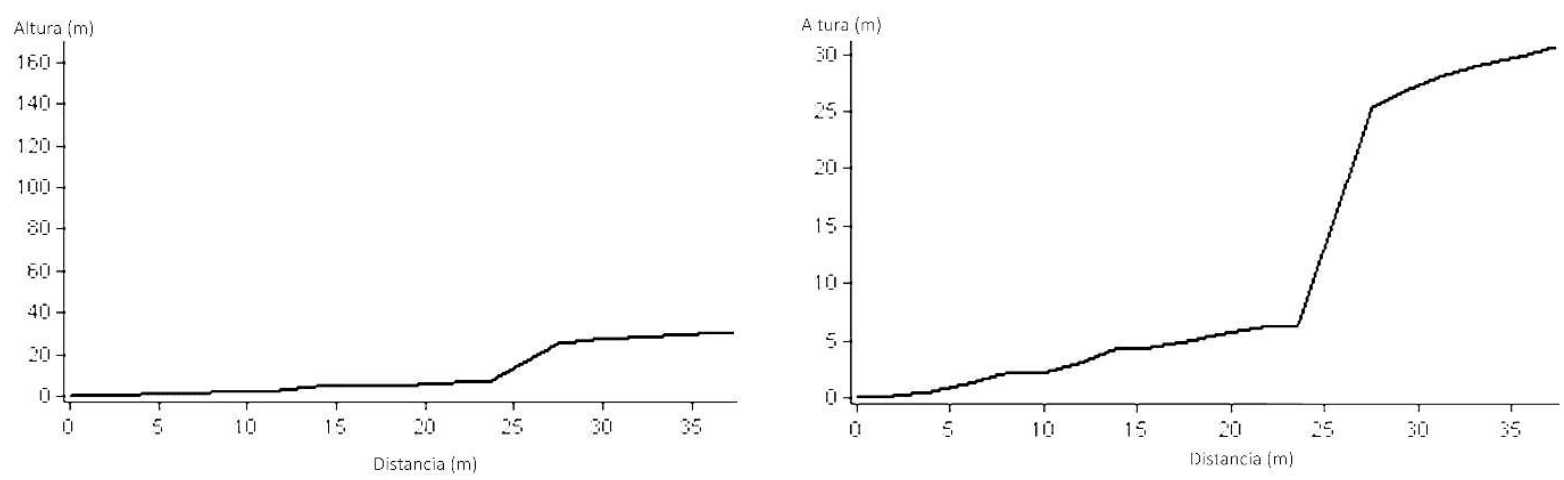

Figura 6 - Perfil tipo de costa 1 (a la izquierda con la altura máxima y a la derecha en detalle).

El tipo 2 de costa está asociado a los acantilados de una mayor altitud, que al igual que en el caso anterior se encuentran en áreas de alta influencia marina. Se trata de los de mayor potencia que se pueden encontrar en el archipiélago y que se pueden considerar como mega-acantilados por su altitud dentro del rango altitudinal en el que se encuentra el archipiélago, donde la altitud máxima alcanza los 192 metros aunque según autores como Bird (2008) muchos de los acantilados que se presentan en esta agrupación quedarían enmarcados dentro de los altos acantilados.

Los depósitos sedimentarios existentes dentro de este tipo quedan reducidos a pequeños sectores de la zona norte, asociados a playas de bloques (coídos) al abrigo de salientes. En el caso de la isla sur este tipo está ligado a pequeñas plataformas litorales de menos de 20 metros. El perfil que se forma en este caso es extremadamente homogéneo, lo que se relaciona con el sustrato predominantemente granítico que presenta una resistencia más homogénea a la erosión que en otros lugares, no existiendo rupturas de pendiente hasta el cliff top, que coincide en ocasiones con la parte más elevada de la isla.

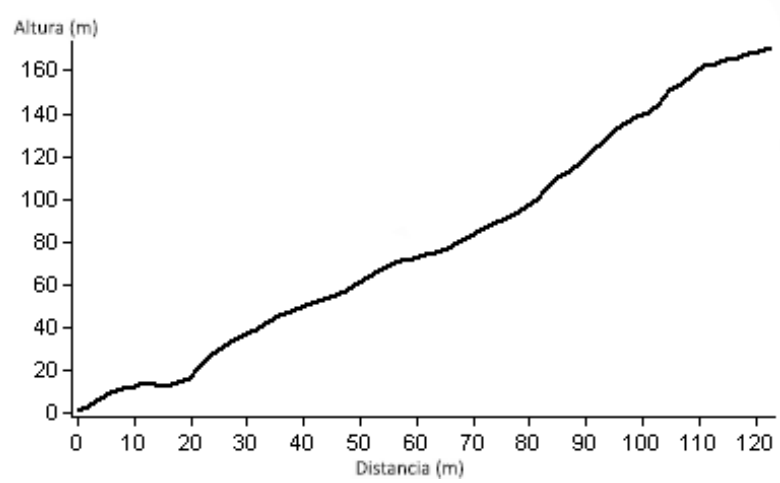

Figura 7 - Perfil topográfico Tipo 2. 
El tipo 3 de costa se caracteriza por un perfil tendido con variaciones en su pendiente en la parte baja en relación con plataformas y zonas de acumulación, que no superan los 100 metros en su desarrollo longitudinal. En las zonas de acumulación hay que destacar la presencia de coídos compuestos por bloques que pueden superar los 5 metros en el eje mayor.
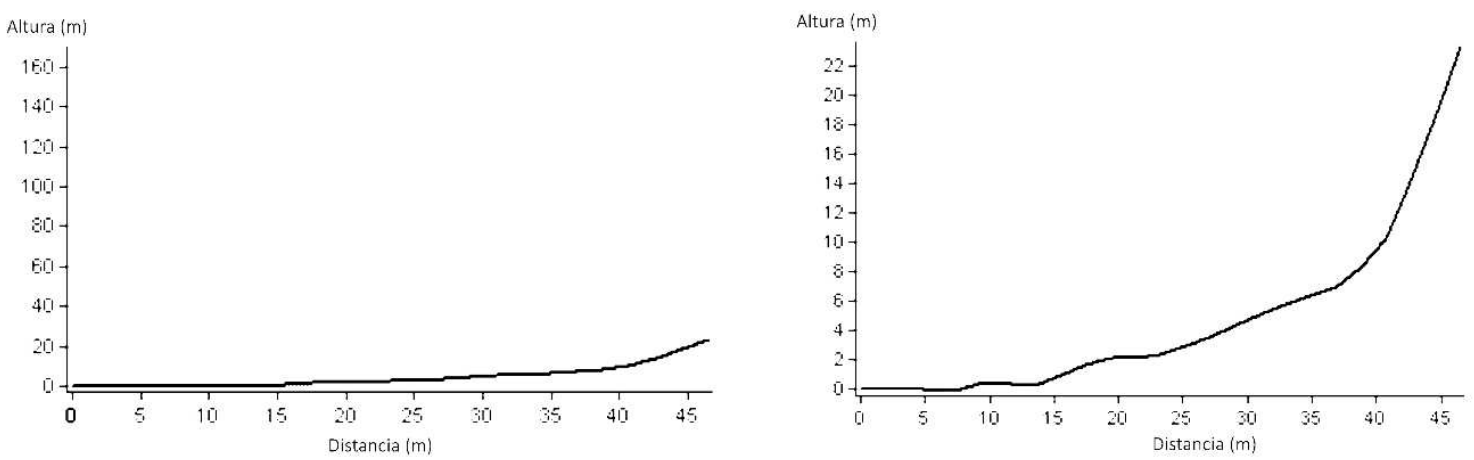

Figura 8 - Perfiles topográficos del tipo 3 (a la izquierda con la altura máxima y a la derecha en detalle).

En el tipo 4 se unen ambas categorías (Tabla 2) dado que sus perfiles presentan una gran similitud, lo que se debe relacionar con la reducida importancia que tiene en este archipiélago la variación altitudinal. Las acumulaciones no son características y tan sólo aparecen representadas con claridad en el extremo sur.
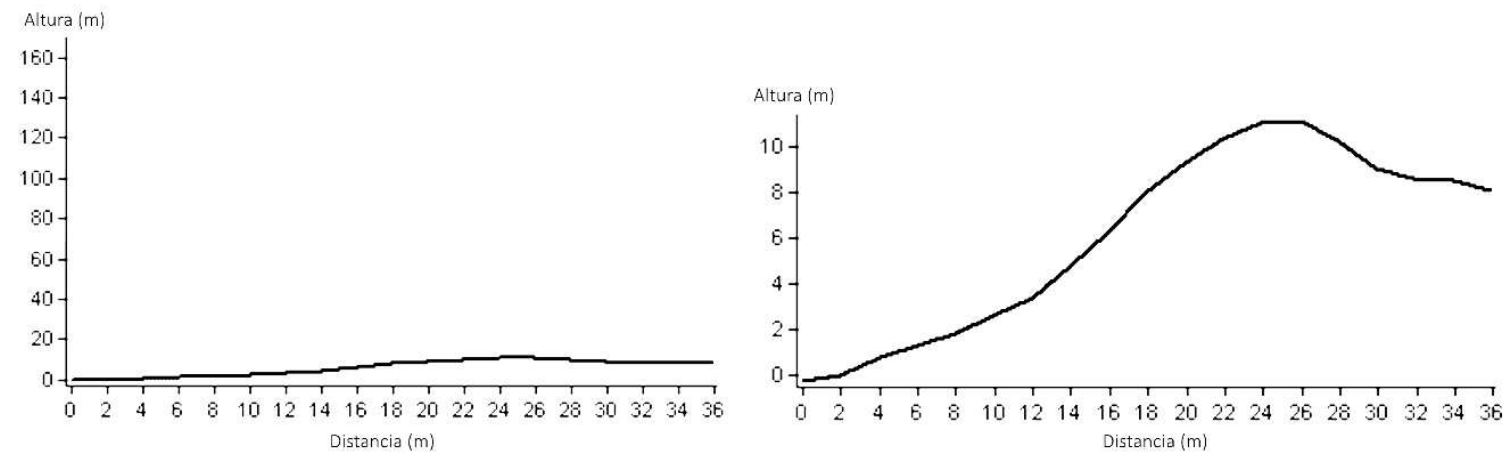

Figura 9 - Perfiles topográficos del tipo 4 (a la izquierda con la altura máxima y a la derecha en detalle).

El tipo 5 de costa se encuentra en relación con plataformas litorales inclinadas en la parte norte. Esta tipología se encuentra en las zonas con una menor importancia de la influencia 
marina, presentando un perfil compuesto por una base casi plana o de pendiente muy baja que evoluciona hacia una ruptura de pendiente relacionada con el fin de la zona de acumulación, a partir de la cual la pendiente aumenta conforme aumenta la altitud.
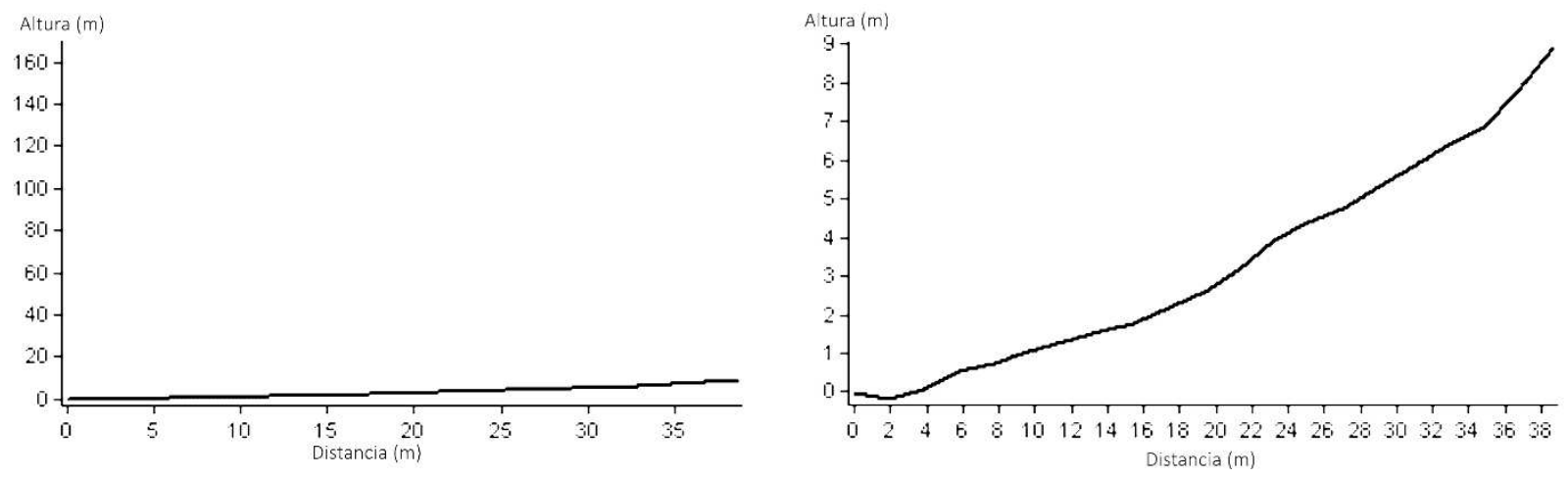

Figura 10 - Perfiles topográficos del tipo 5 (a la izquierda con la altura máxima y a la derecha en detalle).

En el tipo 6 de costa se puede observar un perfil irregular en las proximidades del mar en función de la etapa evolutiva en la que se encuentre la plataforma litoral y que aumenta el porcentaje de pendiente constantemente conforme se aleja de la costa. Se trata de una costa baja con una importante presencia de pequeñas plataformas litorales.
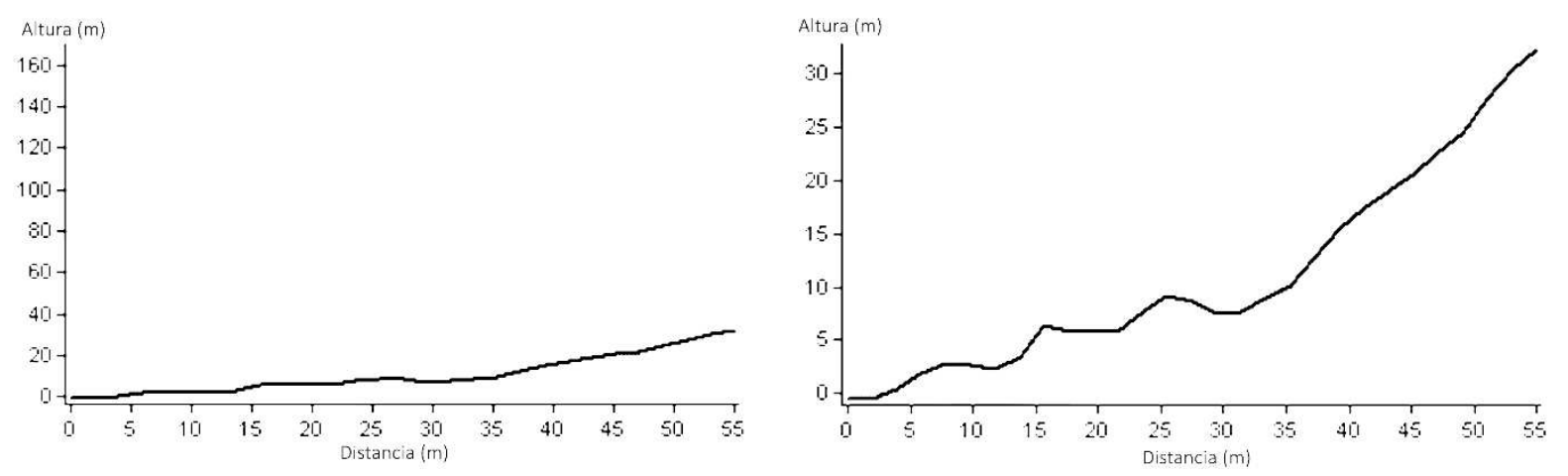

Figura 11 - Perfiles topográficos del tipo 6 (a la izquierda con la altura máxima y a la derecha en detalle).

Los tipos 7 y 8 presentan unas características muy similares, siendo el matiz que lleva a englobarlos en grupos diferentes su contacto con el mar, que es por medio de plataformas litorales en el primer tipo, mientras que en el segundo el contacto es directo. En el tipo 7 su perfil presenta una ruptura de pendiente a partir de la plataforma litoral que puede dar 
lugar a un segundo nivel de plataforma, o zona plana, según su nivel evolutivo derivando posteriormente en una pendiente que se reduce conforme aumenta la altura. Por su parte, el tipo 8 tan sólo aparece en el extremo norte de la vertiente oriental, con una pendiente más homogénea que en el caso anterior, siendo su desarrollo longitudinal mayor que el que del tipo 7, pese a que este está asociado a las plataformas litorales.
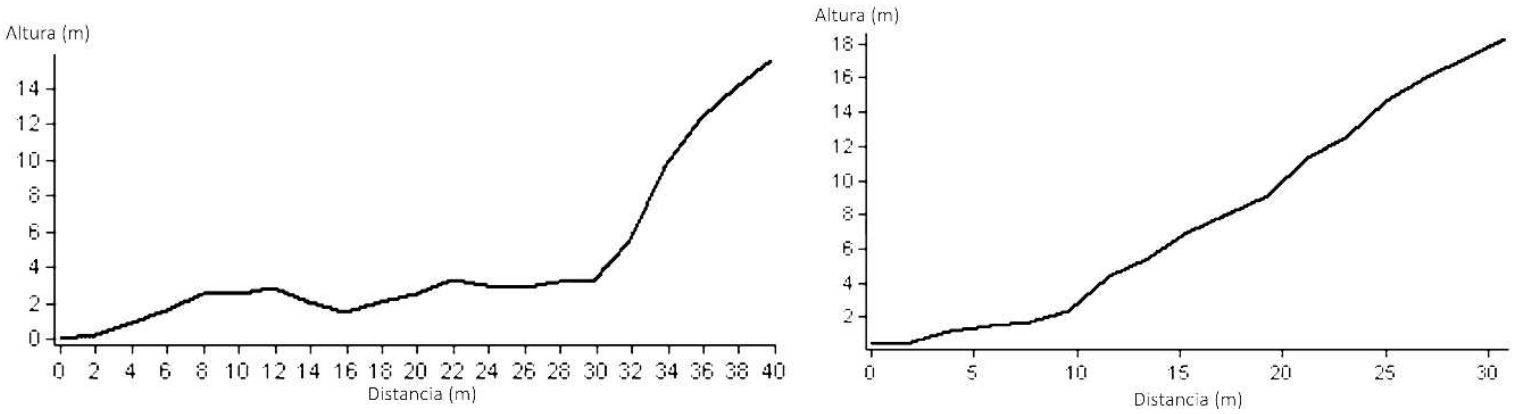

Figura 12 - Perfiles topográficos de detalle, a la izquierda el tipo 7 y a la derecha el tipo 8.

El tipo 9 representa acantilados que entran directamente en el mar y que no tienen ningún tipo de acumulación en su base como consecuencia de la exposición abierta a la influencia marina. En cuanto a su perfil, presenta una pendiente suave en la base, con un posterior cambio brusco en la zona media, para suavizarse progresivamente en la parte superior.
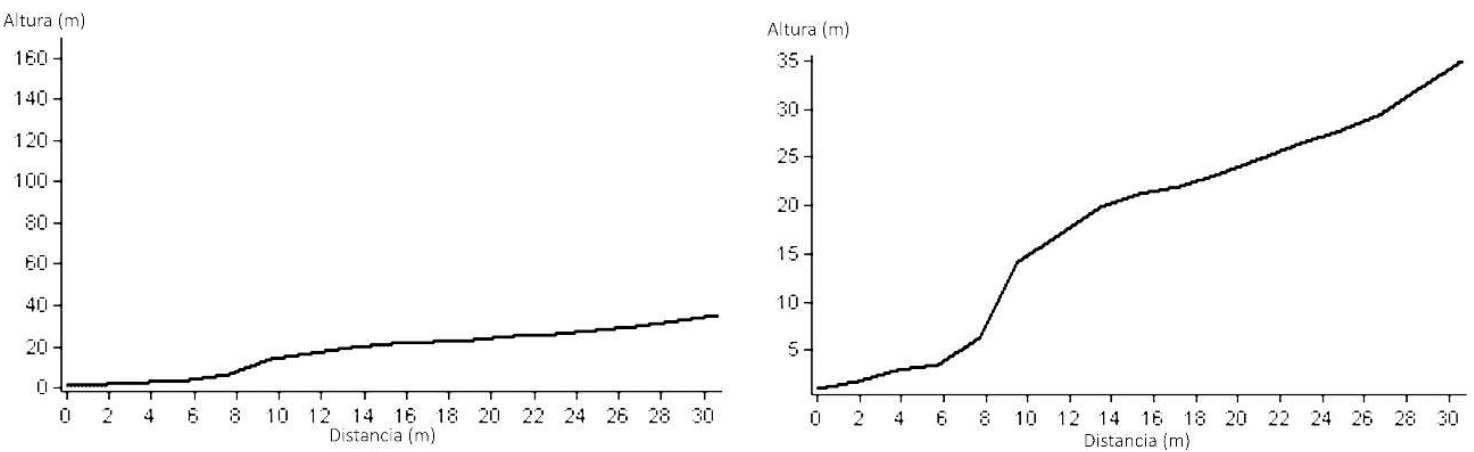

Figura 13 - Perfiles topográficos del tipo 9 (a la izquierda con la altura máxima y a la derecha en detalle).

El tipo 10 que se ha identificado se ha denominado como costa baja, estando limitado a los arenales y pequeñas plataformas como la existente en la trasplaya de Rodas, en el entorno del lagoon. La localización de este tipo está relacionada directamente con las zonas de menor influencia marina, en las que se hace posible la sedimentación de material fino. Se 
trata de una categoría cuyos perfiles topográficos son muy variables al encontrarse en una costa sedimentaria, donde la evolución es mucho más rápida si la comparamos con las costas rocosas (Trenhaile, 1997), por lo que no se puede definir con claridad un perfil tipo para todo el conjunto, pero lo que si se cumple es que las alturas y las pendientes son muy bajas, como se mencionó con anterioridad.

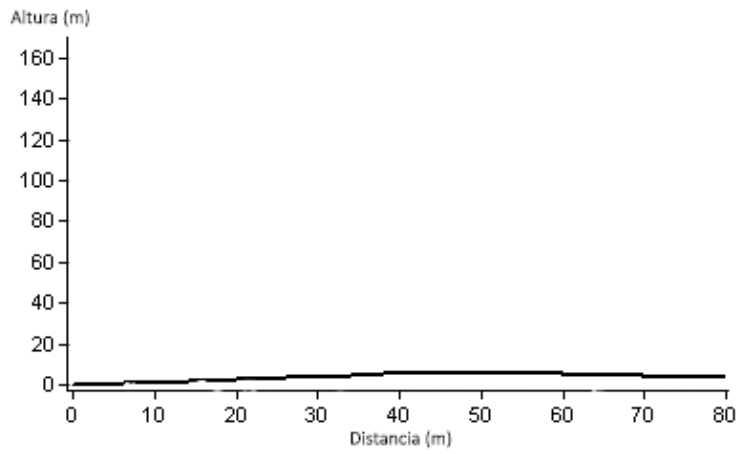

Figura 14 - Perfil topográfico del tipo 10 ajustado a la altura máxima.

\section{Discusión}

Las costas son sistemas dinámicos que están sometidos a una continua evolución (Cowell y Thom, 1994) como consecuencia de los factores que influyen en su dinámica. Dentro de ellos los sectores rocosos presentan una gran originalidad (Trenhaile, 1987; Sunamura, 1992) pero también una gran dificultad a la hora de investigarlos por las dificultades de acceso a muchos lugares y la necesidad para algunos estudios de datos de un amplio espectro temporal.

A lo largo del tiempo se asiste a una continua evolución de los métodos empleados para conocer el funcionamiento de los sistemas costeros y su evolución. En el caso de las costas rocosas los esfuerzos se han centrado, por una parte, en mejorar el conocimiento mediante modelos matemáticos (Trenhaile, 2000; Trenhaile, 2001a; Trenhaile, 2001b; Trenhaile, 2001c; Trenhaile, 2004b; Trenhaile, 2005; Trenhaile, 2010a) para simular, por ejemplo, los diferentes escenarios de evolución de las plataformas litorales, con el objetivo de entender y lograr tipificar escenarios prospectivos del comportamiento de los sistemas costeros rocosos o, por otro lado, para conocer el grado de movilidad de los acantilados (Epifanio et 
al., 2013; Marqués et al., 2011), de las playas de bloques asociadas (Pérez-Alberti y Trenhaile, 2015a; Pérez-Alberti y Trenhaile, 2015b) o centrarse en el estudio del papel de las herencias (Trenhaile et al., 1999) quedando en un segundo plano las clasificaciones tipológicas de las costas realizadas desde un punto de vista más genérico (Finkl, 2004; Balaguer et al., 2006).

En el momento actual, con el avance de las tecnologías SIG, se pueden lograr unas clasificaciones con una gran precisión. En este punto también es muy importante destacar la accesibilidad que existe a los datos necesarios para realizar estudios de este tipo a nivel de detalle, dado que toda la información utilizada en la realización de este estudio es accesible de forma gratuita a través del Centro de Descargas del Instituto Geográfico Nacional de España.

Los trabajos existentes para el archipiélago de las Illas Cíes se han centrado casi en su totalidad, exceptuando las guías del parque nacional que no profundizan en la dinámica geomorfológica, en el sistema playa-duna-lagoon de Rodas (Costas et al., 2005; Costas et al., 2006a; Costas et al., 2006b; Costas et al., 2006c; Costas y Alejo, 2007; Costas, 2008; Costas et al., 2009) que focaliza la atención de los estudiosos dada su importancia no solo como atractivo turístico sino porque también se busca conocer la dinámica de la zona y su futura evolución para tratar de solventar los problemas que se le pueden avecinar como la colmatación del lagoon.

Por ello, con esta investigación se trató de dar un paso más en el conocimiento de las islas y realizar una caracterización pormenorizada de las características de su costa para de este modo conseguir un mayor conocimiento de cada zona y poder comprender como ha sido su evolución pasada y cual será, a priori, la dinámica futura que va a seguir este archipiélago.

Conocer los rasgos topográficos de la fachada costera ayuda a la comprensión de su dinámica evolutiva, al tiempo que aporta una cartografía que potencia el acercamiento a los ecosistemas. El conocimiento y clasificación de la costa del archipiélago da pie a un mejor entendimiento del comportamiento de cada sector y permite evaluar de una forma más clara los agentes que posibilitan las distintas características dominantes en cada zona. Los mismos agentes que se pueden encontrar en este caso de estudio como es la influencia marítima, el aumento del nivel del mar, los vientos o las precipitaciones, tienen igualmente 
importancia en otras zonas en las que dicha caracterización de tipologías costeras puede llevar a una mejor comprensión de los inconvenientes que suscita la localización de infraestructuras o asentamientos humanos. De este modo mediante el entendimiento del porqué da las distintas formas que se pueden encontrar en el litoral se lograría un mejor planeamiento y se evitarían riesgos, de ahí la importancia de lograr una metodología sencilla y clara para la clasificación de las zonas costeras. Gracias a estas metodologías se puede llegar a una rápida caracterización de sectores litorales diversos, favoreciendo de este modo una mayor rapidez a la hora de llevar a cabo actuaciones en la franja costera y pudiendo posibilitar una ordenación del litoral más sencilla.

\section{Conclusiones}

El estudio que se ha presentado permite concluir lo siguiente:

1. La gran importancia que tienen las herramientas SIG a la hora de poder caracterizar las costas delimitando tipologías, suponiendo en todo caso una importante automatización de los procesos y permitiendo llegar a caracterizaciones de gran complejidad al permitir utilizar un gran número de variables y reduciendo en buena medida los tiempos de trabajo.

2. Los métodos empleados para la clasificación costera de las Illas Cíes puede ser extrapolable a otros sectores costeros, en los que incluso se podría aumentar el número de variables utilizadas en este caso de estudio, como podría ser la inclusión de la litología en aquellas zonas en las que el sustrato variara, lo que no ocurría en el caso de las Cíes.

3. Se han diferenciado claramente las dos vertientes del archipiélago, lo que era de esperar al utilizar variables como la pendiente y la altitud y estando los valores más elevados de estas en la vertiente occidental, donde se superan los 180 metros y se alcanzan pendientes de $80^{\circ}$.

4. La combinación de los tipos de costa con otras capas de información, como suelos, flora o fauna, puede ser de gran utilidad a la hora de entender los ecosistemas desarrollados sobre costas rocosas. 


\section{Referencias}

BALAGUER, P., et al., 2006. Zonificación del litoral balear frente a un posible derrame o vertido de hidrocarburo. Establecimiento de un Índice de Sensibilidad Ambiental (ISA) de la línea de costa. In: A. PÉREZALBERTI and J. LÓPEZ-BEDOYA eds., Actas de la IX Reunión Nacional de Geomorfología, Santiago de Compostela, pp. 311-322.

BIRD, E.C.F., 2008. Coastal Geomorphology. 2. ed. ed. Chichester [u.a.]: Wiley ISBN 9780470517291.

CARBALleIRA, A., et al. Bioclimatología de Galicia. A Coruña: Fundación P. Barrié de la Maza. Conde de Fenosa, 1983.

CASAL VILA, B., 2003. Gran Enciclopedia Galega Silveiro Cañada. Lugo: El Progreso.

CHUVIECO, E., 2010. Teledetección Ambiental: La Observación De La Tierra Desde El Espacio. Barcelona: Ariel.

CONRAD, O., et al, 2015. System for Automated Geoscientific Analyses (SAGA) V. 2.1.4. Geoscientific Model Development, July 1 , vol. 8 , no. 7, pp. 1991-2007. Available from: https://doaj.org/article/ab5da2a980594222ad31bc4c7a3fdfe1 ISSN 1991-959X. DOI 10.5194/gmd-81991-2015.

COSTAS, S., 2008. Origen y evolución del conjunto playa-duna-"lagoon" de Cíes (Parque Nacional MarítimoTerrestre De Las Islas Atlánticas De Galicia). Madrid: Organismo Autónomo Parques Nacionales, D.L.

COSTAS, S. y ALEJO, I., 2007. Local and Global Influences on the Evolution of a Transgressive Sand Barrier: Cíes Barrier, Northwest Spain. Journal of Coastal Research, no. 50, pp. 1121-1125.

COSTAS, S., ALEJO, I. y NOMBELA, M.A., 2006a. Sand barrier behavior under man-induced inlet relocation. Journal of Coastal Research, January 1, pp. 428-432. Available from: http://www.jstor.org/stable/25741610 ISSN 0749-0208.

COSTAS, S., ALEJO, I. y NOMBELA, M.A., 2006b. The Role of Sediment Source in Beach Management. A Case Study from Cíes Islands (NW Spain). Journal of Coastal Research, January 1, pp. 1389-1394. Available from: http://www.jstor.org/stable/25742982 ISSN 0749-0208.

COSTAS, S., ALEJO, I., VILA-CONCEJO, A. y NOMBELA, M.A., 2005. Persistence of Storm-Induced Morphology on a Modal Low-Energy Beach: A Case Study from NW-Iberian Peninsula. Marine Geology, vol. 224, no. 1, pp. 4356. Available from: http://www.sciencedirect.com/science/article/pii/S0025322705002641 CrossRef. ISSN 0025-3227. DOI 10.1016/j.margeo.2005.08.003.

COSTAS, S., SOBRINO, C.M., ALEJO, I. y PÉREZ-ARLUCEA, M., 2009. Holocene Evolution of a Rock-Bounded Barrier-Lagoon System, Cíes Islands, Northwest Iberia. Earth Surface Processes and Landforms, September 15, vol. 34, no. 11, pp. 1575-1586 CrossRef. ISSN 0197-9337. DOI 10.1002/esp.1849.

COSTAS, S., et al, 2006c. Cyclical Evolution of a Modern Transgressive Sand Barrier in Northwestern Spain Elucidated by GPR and Aerial Photos. Journal of Sedimentary Research, September 1, vol. 76, no. 9, pp. 10771092. Available from: http://jsedres.geoscienceworld.org/cgi/content/abstract/76/9/1077 CrossRef. ISSN 1527-1404. DOI 10.2110/jsr.2006.094.

COWELL, P.J. y THOM, B.G., 1994. Morphodynamics of coastal evolution. In: Cambridge: Cambridge University Press. Morphodynamics of Coastal Evolution, pp. 33-86.

D'ERCOLE, R., et al. Recursos De Respuesta Inmediata Y De Recuperación Temprana Ante La Ocurrencia De Un Sismo Y/O Tsunami En Lima Metropolitana Y Callao. HAL CCSD, 2011 Available from: https://hal.archivesouvertes.fr/halsde-00964437.

DEL RíO, L. y GRACIA, F.J., 2007. Análisis de la vulnerabilidad de los acantilados atlánticos de la provincia de Cádiz ante la erosión costera. Cuaternario Y Geomorfología, vol. 21 (1), pp. 87-101. 
DEL RíO, L. y GRACIA, F.J., 2009. Erosion Risk Assessment of Active Coastal Cliffs in Temperate Environments.

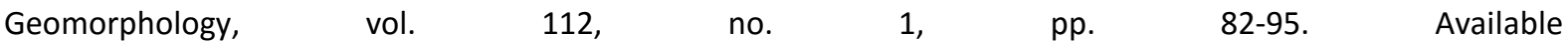
from: http://www.sciencedirect.com/science/article/pii/S0169555X09002165 CrossRef. ISSN 0169-555X. DOI 10.1016/j.geomorph.2009.05.009.

DEL RíO, L., GRACIA, F.J. y BENAVENTE, J., 2009. Mass Movements and Cliff Retreat Along the SW Spanish Coast. Journal of Coastal Research, January 1, vol. SI, no. 56, pp. 717-721. Available from: http://www.jstor.org/stable/25737672 ProQuest Environmental Science Journals. ISSN 0749-0208.

DIKAU, R., 1989. The Application of a Digital Relief Model to Landform Analysis in Geomorphology. Three Dimensional Applications in Geographical Information Systems, pp. 51-77.

EBERT, K., HÄTTESTRAND, C., HALL, A.M. y ALM, G., 2011. DEM Identification of Macroscale Stepped Relief in Artic Northern Sweden. Geomorphology, vol. 123(3), pp. 339-350.

EMERY, K.O. y KUHN, G.G., 1982. Sea Cliffs: Their Processes, Profiles, and Classification. GSA Bulletin, July 1,

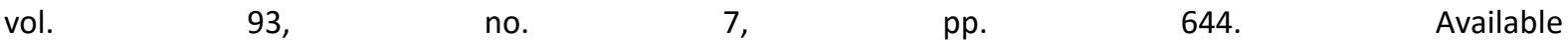
from: http://bulletin.geoscienceworld.org/cgi/content/abstract/93/7/644 CrossRef. ISSN 0016-7606. DOI SCTPPA>2.0.CO;2.

EPIFÂNIO, B., ZÊZERE, J.L. y NEVES, M., 2013. Identification of Hazardous Zones Combining Cliff Retreat Rates with Landslide Susceptibility Assessment. Journal of Coastal Research, January 1, vol. 2, no. 65, pp. 1681. Available from: http://search.proquest.com/docview/1681906535 ProQuest Environmental Science Journals. ISSN 0749-0208.

FERRARIS, F., FIRPO, M. y PAZZAGLIA, F.J., 2012. DEM Analyses and Morphotectonic Interpretation: The PlioQuaternary Evolution of the Eastern Ligurian Alps, Italy. Geomorphology, May 1, vol. 149, pp. 27-40. Available from: http://www.sciencedirect.com/science/article/pii/S0169555X12000232 CrossRef. ISSN 0169-555X. DOI 10.1016/j.geomorph.2012.01.009.

FINKL, C.W., 2004. Coastal Classification: Systematic Approaches to Consider in the Development of a Comprehensive Scheme. Journal of Coastal Research, January 1, vol. 20, no. 1, pp. 166-213. Available from: http://www.jstor.org/stable/4299277CrossRef. ISSN 0749-0208. DOI CCSATC]2.0.CO;2.

GAO, M., et al, 2013. DEM and GIS Analysis of Geomorphic Indices for Evaluating Recent Uplift of the Northeastern Margin of the Tibetan Plateau, China. Geomorphology, May, vol. 190, pp. 61-72 CrossRef. ISSN 0169-555X. DOI 10.1016/j.geomorph.2013.02.008.

GREEN, D.R. y KING, S.D., 2003. Coastal and Marine Geo-Information Systems : Applying the Technology to the Environment. Dordrecht: Kluwer Academic Publishers ISBN 9780306480027.

IPCC., 2014. Climate Change 2013. Cambridge University Press, March 1 ISBN 110705799X.

KASAI, M., IKEDA, M., ASAHINA, T. y FUJISAWA, K., 2009. LiDAR-Derived DEM Evaluation of Deep-Seated Landslides in a Steep and Rocky Region of Japan. Geomorphology, vol. 113, no. 1, pp. 57-69. Available from: http://www.sciencedirect.com/science/article/pii/S0169555X09002359 CrossRef. ISSN 0169-555X. DOI 10.1016/j.geomorph.2009.06.004.

KAWABATA, D. y BANDIBAS, J., 2009. Landslide Susceptibility Mapping using Geological Data, a DEM from ASTER Images and an Artificial Neural Network (ANN). Geomorphology, vol. 113, no. 1, pp. 97-109. Available from: http://www.sciencedirect.com/science/article/pii/S0169555X09002372 CrossRef. ISSN 0169-555X. DOI 10.1016/j.geomorph.2009.06.006.

LIN, Z. y OGUCHI, T., 2006. DEM Analysis on Longitudinal and Transverse Profiles of Steep Mountainous Watersheds. Geomorphology, vol. 78, no. 1, pp. 77-89. Available from: http://www.sciencedirect.com/science/article/pii/S0169555X06000304CrossRef. ISSN 0169-555X. DOI 10.1016/j.geomorph.2006.01.017.

MARQUES, F., MATILDES, R. y REDWEIK, P., 2011. Statistically Based Sea Cliff Instability Hazard Assessment of Burgau-Lagos Coastal Section (Algarve, Portugal). Journal of Coastal Research, January 1, vol. SI, no. 64, pp. 927. Available from: http://search.proquest.com/docview/1675866869 ProQuest Environmental Science Journals. ISSN 0749-0208. 
MOURIÑO LOURIDO, J., OTERO PÉREZ, X.L., SIERRA-ABRAÍN, F. y ALONSO IGLESIAS, P., 2002. Guía Das Illas Cíes. Vigo: Concello de Vigo.

NELSON, J.R., et al., 2015 Approach for Assessing Coastal Vulnerability to Oil Spills for Prevention and Readiness using GIS and the Blowout and Spill Occurrence Model. Ocean \& Coastal Management, 2015, vol. 112. pp. 1-11. DOI 10.1016/j.ocecoaman.2015.04.014

PANNEKOEK, A.J., 1970. Aditional Geomorphological Data on the Ria Area of Western Galicia (Spain). Leidse Geologische Modelingen, no. 37, pp. 185-194.

PEREIRA, A., GOMES, A. y PEREZ-AlBERTI, A., 2013. Vantagens Da Utilização De Dados ALS (LiDAR) Na Caracterização Morfológica Das Vertentes Costeiras Da Galiza (Espanha): Aplicação Ao Setor De Mougás. GOT Geography and Spatial Planning Journal, December, vol. 4, pp. 223-248 CrossRef. DOI 10.17127/got/2013.4.010.

PÉREZ-ALBERTI, A., 1986. Xeografía De Galicia. Vigo: Galaxia.

PÉREZ-ALBERTI, A., 2014. Caracterización Geomorfológica Mediante SIG De La Costa Septentrional de Marruecos. Geo-Temas, vol. 14, pp. 59-62 ISSN 1576-5172.

PÉREZ-ALBERTI, A. y TRENHAILE, A.S., 2015a. An Initial Evaluation of Drone-based Monitoring of Boulder Beaches in Galicia, North-western Spain. Earth Surface Processes and Landforms, January, vol. 40, no. 1, pp. 105-111. Available from: http://onlinelibrary.wiley.com/doi/10.1002/esp.3654/abstract ISSN 0197-9337. DOI 10.1002/esp.3654.

PÉREZ-ALBERTI, A. y TRENHAILE, A.S., 2015b. Clast Mobility within Boulder Beaches Over Two Winters in Galicia, Northwestern Spain. Geomorphology, November, vol. 248, pp. 411-426 CrossRef. ISSN 0169-555X. DOI 10.1016/j.geomorph.2015.08.001.

PÉREZ-ALBERTI, A., CASAIS, M. y BLANCO-CHAO, R., 2002. Stability of Sedimentary Cliffs in the Coast of Galicia (NW Spain): Long Term Inheritance Influence in Rocky Coastal Systems. The Changing Coast Eds. Littoral EUROCOAST, pp. 281-285.

PÉREZ-ALBERTI, A., PIRES, A., FREITAS, L. y CHAMINÉ, H., 2011. GIS mapping and shoreline change analysis along the rocky coast of Galicia (NW Spain): preliminar approach. Institution of Civil Engineers Book of Abstracts, 7th Conference on Coastal Management, Innovative Coastal Zone Management: Sustentainable Engineering for a Dynamic Coast, Belfast. ICE Publishing, London, UK, pp. 262-263.

PÉREZ-ALBERTI, A., PIRES, A., FREITAS, L. y CHAMINÉ, H., 2013. Shoreline Change Mapping Along the Coast of Galicia, Spain. Proceedings of the Institution of Civil Engineers, September 1, vol. 166, no. 3, pp. 125. Available from: http://search.proquest.com/docview/1470864973 ISSN 1741-7597.

PEREZ-ALBERTI, A., et al, 2012. The Effect of Boulders on Shore Platform Development and Morphology in Galicia, North West Spain. Continental Shelf Research, October 1, vol. 48, pp. 122 ISSN 0278-4343. DOI 10.1016/j.csr.2012.07.014.

POLGalicia., 2010. Plan De Ordenación do Litoral De Galicia. Santiago de Compostela: Consellaría de Medio Ambiente, Territorio e Infraestruturas.

ROSÓN, G., CABANAS, J.M. y PÉREZ, F.F., 2008. Hidrografía Y Dinámica De La Ría de Vigo: Un Sistema De Afloramiento.

SUNAMURA, T., 1992. Geomorphology of Rocky Coasts. Chichester: Wiley ISBN 0471917753.

TERRANOVA, O., ANTRONICO, L., COSCARELLI, R. y IAQUINTA, P. Soil Erosion Risk Scenarios in the Mediterranean Environment using RUSLE and GIS: An Application Model for Calabria (Southern Italy). Geomorphology, 2009, vol. 112, no. 3. pp. 228-245. Available from: http://www.sciencedirect.com/science/article/pii/S0169555X09002487 CrossRef. ISSN 0169-555X. DOI 10.1016/j.geomorph.2009.06.009.

TRENHAILE, A.S., 1987. The Geomorphology of Rock Coasts. England: ISBN 0198232799.

TRENHAILE, A.S., 1997. Coastal Dynamics and Landforms. Oxford: Clarendon Press ISBN 0198233531. 
TRENHAILE, A.S., 2000. Modeling the Development of Wave-Cut Shore Platforms. Marine Geology, vol. 166, no. 1, pp. 163-178. Available from: http://www.sciencedirect.com/science/article/pii/S002532270000013X CrossRef. ISSN 0025-3227. DOI 10.1016/S0025-3227(00)00013-X.

TRENHAILE, A.S., 2001a. Modeling the Effect of Late Quaternary Interglacial Sea Levels on Wave-Cut Shoreplatforms. Marine Geology, vol. 172, pp. 205-223.

TRENHAILE, A.S., 2001b. Modelling the Quaternary Evolution of Shore Platforms and Erosional Continental Shelves. Earth Surface Processes and Landforms, September, vol. 26, no. 10, pp. 1103-1128. Available from: http://onlinelibrary.wiley.com/doi/10.1002/esp.255/abstract CrossRef. ISSN 0197-9337. DOI 10.1002/esp.255.

TRENHAILE, A.S., 2002a. Rock Coasts, with Particular Emphasis on Shore Platforms. Geomorphology, vol. 48, no. 1, pp. 7-22. Available from: http://www.sciencedirect.com/science/article/pii/S0169555X02001733 CrossRef. ISSN 0169-555X. DOI 10.1016/S0169-555X(02)00173-3.

TRENHAILE, A.S., 2002b. Modeling the Development of Marine Terraces on Tectonically Mobile Rock Coasts. Marine Geology, vol. 185, no. 3, pp. 341-361. Available from: http://www.sciencedirect.com/science/article/pii/S0025322702001871 CrossRef. ISSN 0025-3227. DOI 10.1016/S0025-3227(02)00187-1.

TRENHAILE, A.S., 2003. Chapter 14 Modeling shore platforms: present status and future developments. In: V.C. LAKHAN ed., Advances in Coastal ModelingÁmsterdam: Elsevier B.V. Chapter 14 Modeling Shore Platforms: Present Status and Future Developments, pp. 393-409 ISBN 0444511490. DOI 10.1016/S0422-9894(03)801319.

TRENHAILE, A.S., 2004. Modeling the Effect of Tidal Wetting and Drying on Shore Platform Development. Journal of Coastal Research, vol. 20, no. 4, pp. 1049-1060. Available from: http://www.bioone.org/doi/full/10.2112/03-0002R.1 CrossRef. ISSN 0749-0208. DOI 10.2112/03-0002R.1.

TRENHAILE, A.S., 2005. Modelling the Effect of Waves, Weathering and Beach Development on Shore Platform Development. Earth Surface Processes and Landforms, May, vol. 30, no. 5, pp. 613-634. Available from: http://onlinelibrary.wiley.com/doi/10.1002/esp.1166/abstract CrossRef. ISSN 0197-9337. DOI 10.1002/esp.1166.

TRENHAILE, A.S., et al, 1999. Rock Coast Inheritance: An Example from Galicia, Northwestern Spain. Earth Surface Processes and Landforms, July, vol. 24, no. 7, pp. 605-621. Available from: http://onlinelibrary.wiley.com/doi/10.1002/(SICI)1096-9837(199907)24:7<605::AID-ESP977>3.0.CO;21/abstract CrossRef. ISSN 0197-9337. DOI AID-ESP977>3.0.CO;2-1. 